\title{
PROMOVIENDO LA AUTODETERMINACIÓN EN EL AULA: EL MODELO \\ DE ENSENAANZA Y APRENDIZAJE DE LA AUTODETERMINACIÓN ${ }^{1}$
}

\section{Promoting self-determination skills in the classroom: the Self-determined Learning Model of Instruction (Spanish version)}

Cristina Mumbardó-Adam

Universitat Ramon Llull. Facultat de Psicologia, Ciències de l'Educació i de l'Esport Blanquerna. C/ Císter, 34. 08022 Barcelona

cristinama6@blanquerna.url.edu

Eva Vicente SÁNCHEZ

Universidad de Zaragoza. Facultad de Educación

Climent Giné i Giné

Universitat Ramon Llull. Facultat de Psicologia, Ciències de l'Educació i de l'Esport Blanquerna

Joan Guardia-Olmos

Universitat de Barcelona. Facultat de Psicologia. Institut de Neurociències

Sheida K. RALEY

University of Kansas. Beach Center on Disability and Kansas University Center on Developmental Disabilities

Miguel Ángel Verdugo Alonso

Universidad de Salamanca. Facultad de Psicologia

Recepción: 6 de diciembre de 2016

Aceptación definitiva: 6 de abril de 2017

1 Este trabajo ha sido financiado por el SUR de la DEC de la Generalitat de Catalunya gracias a una beca pre-doctoral [2016FI_B00253] y por los Fondos Sociales Europeos.

(C) Ediciones Universidad de Salamanca / CC BY-NC-ND

Siglo Cero, vol. 48 (2), n. ${ }^{\circ}$ 262, 2017, abril-junio, pp. 41-59 
PROMOVIENDO LA AUTODETERMINACIÓN EN EL AULA:

EL MODELO DE ENSEÑANZA Y APRENDIZAJE DE LA AUTODETERMINACIÓN

C. MUMBARDÓ-ADAM, E. VICENTE, C. GINÉ I GINÉ, J. GUARDIA-OLMOS, S. K. RALEY Y M. Á. VERDUGO

RESUMEN: La promoción de la autodeterminación en el ámbito escolar sigue siendo una tarea pendiente en el contexto español, a pesar de la existencia de diferentes instrumentos orientados a la enseñanza de habilidades relacionadas con la conducta autodeterminada. Algunos instrumentos, como el Self-Determined Learning Model of Instruction o Modelo de Enseñanza/Aprendizaje de la Autodeterminación, han demostrado su eficacia en el fomento de dichas habilidades en contexto estadounidense. El presente estudio pretende dar a conocer la versión adaptada al contexto español de dicho modelo de intervención, su modo de implementación, así como presentar las características y las evidencias empíricas de los estudios que avalan su eficacia, a fin de ofrecer un instrumento de intervención que los profesionales educativos puedan usar en el ámbito escolar.

Palabras Clave: autodeterminación; intervención; formación de profesionales educativos.

AвSTRAct: Within Spanish context, initiatives to promote self-determination in educational settings still lacking despite of the availability of instruments designed to enable the instruction of self-determination skills, such as the Self-Determined Learning Model of Instruction. This evidence-based practice enables teachers to instruct students to develop self-determination actions and skills. This study aims to present the Spanish translated and adapted version of the afore mentioned program/tool, in an effort to improve the focus of self-determination instruction in the Spanish educational context by providing practitioners with a model of instruction intended to teach skills associated with the promotion and enhancement of self-determined action.

KEYWORDS: self-determination; intervention tool; teacher education.

\section{Introducción}

P ROMOVER ESTRATEGIAS RELACIONADAS CON LA AUTODETERMINACIÓN en el contexto escolar ha sido objeto de estudio en el ámbito educativo y, en concreto, en el de las necesidades educativas especiales, y se ha erigido como estrategia clave para la enseñanza de habilidades relacionadas con una transición a la vida adulta de calidad (Shogren, Wehmeyer, Palmer, Rifenbark y Little, 2015; Test, Mazzotti, Mustian, Fowler, Kortering y Kohler, 2009). Aunque los beneficios de la enseñanza de estrategias relacionadas con la autodeterminación se han descrito principalmente en alumnos con discapacidad (p. e., Agran, Cavin, Wehmeyer y Palmer, 2010), la investigación pone de manifiesto su relevancia para todos los alumnos, con y sin discapacidad (Shogren, Lopez, Wehmeyer, Little y Pressgrove, 2006).

En este sentido, recientemente se ha revisado el concepto de autodeterminación vigente hasta el momento bajo el Modelo Funcional de Autodeterminación (Wehmeyer, 2003), redefiniendo el constructo bajo el prisma de la Teoría de la Agencia Causal (Shogren, Wehmeyer, Palmer, Forber-Pratt, Little y López, 2015). Este nuevo marco teórico define la autodeterminación como "una característica disposicional que se pone de manifiesto cuando la persona actúa como agente causal de su propia vida” (Shogren et al., 2015: 258). Definir la autodeterminación como característica 
PROMOVIENDO LA AUTODETERMINACIÓN EN EL AULA:

EL MODELO DE ENSEÑANZA Y APRENDIZAJE DE LA AUTODETERMINACIÓN

C. MUMBARDÓ-ADAM, E. VICENTE, C. GINÉ I GINÉ, J. GUARDIA-OLMOS, S. K. RALEY Y M. Á. VERDUGO

disposicional implica reconocer unas características estables a toda acción que se pueda definir como autodeterminada. En concreto, tres son las dimensiones o características esenciales que definen una acción como autodeterminada: (1) volición, (2) agencialidad y (3) creencias sobre las acciones y el control ejercido (Shogren et al., 2015). Actuar de forma volitiva implica hacerlo con autonomía, eligiendo objetivos basados en las propias preferencias e iniciando las acciones cuando y como uno escoge hacerlo. Por otro lado, las acciones agenciales se definen como la capacidad de autorregular las propias acciones, para avanzar hacia la consecución de los objetivos fijados, monitoreando y autosupervisando este proceso (Palmer, Wehmeyer y Shogren, 2016) mediante el uso de la autorregulación, la autodirección y el pensar en vías alternativas de acción. Por último, las creencias de la persona sobre el control que ejerce sobre sus acciones son el conjunto de conocimientos que uno alberga sobre las propias capacidades y las habilidades que se requieren para alcanzar sus objetivos. Estas creencias se construyen mediante las expectativas de control que uno tiene, la capacitación psicológica o empoderamiento que uno ha ido construyendo en base a experiencias previas y la capacidad autorrealizadora que las acciones tienen para la persona. Parece pues indiscutible que cualquier persona puede desarrollar acciones autodeterminadas siempre que disponga de las habilidades, los conocimientos, los apoyos y las oportunidades para hacerlo. Estas características esenciales se desarrollan a través de la adquisición y oportunidades de aprendizaje de múltiples componentes estrechamente relacionados (p. e., resolución de problemas, establecimiento de metas, toma de decisiones y autoevaluación y monitorización).

Así, la Teoría de la Agencia Causal (Shogren et al., 2015) proporciona un marco idóneo para la evaluación y la promoción de la autodeterminación para todos los alumnos, con o sin discapacidad, en todos sus contextos de desarrollo. Los recursos evaluativos se convierten en un elemento de vital importancia como paso fundamental en cualquier proceso de enseñanza. Dichos recursos permiten identificar las necesidades de la persona y guiar la toma de decisiones para el establecimiento de un programa instruccional específico en un ámbito determinado. A su vez, estas herramientas de evaluación proporcionan una medida inicial, de evaluación continua y/o final a lo largo de la implantación de un programa de instrucción, a fin de determinar la eficacia del mismo y detectar posibles áreas de mejora (Vicente, Guillén y Verdugo, 2014). Relacionado con el Modelo Funcional de Autodeterminación (Wehmeyer, 2003) se han propuesto distintos instrumentos de evaluación: el Arc's Self-Determination Scale (SDS; Wehmeyer y Kelchner, 1995), que ha sido adaptado al español por diversos grupos de investigación (p. e., Wehmeyer, Peralta, Zulueta, González y Sobrino, 2006) y la Escala ARC-INICO de evaluación de la autodeterminación, que basándose en el instrumento anterior se ha desarrollado recientemente en España (Verdugo et al., 2015). Además, el Self-Determination Inventory: Student Report (Shogren, Wehmeyer, Little, Forber-Pratt, Palmer y Seo, 2015) constituye la primera iniciativa evaluativa desarrollada bajo el actual prisma teórico y que está siendo validado y adaptado también al español. No obstante, si bien se dispone ya en contexto español de instrumentos validados para una rigurosa evaluación de la autodeterminación, debería prestarse mayor atención y recursos a dotar a los profesionales

(C) Ediciones Universidad de Salamanca / CC BY-NC-ND

Siglo Cero, vol. 48 (2), n. ${ }^{\circ}$ 262, 2017, abril-junio, pp. 41-59 
PROMOVIENDO LA AUTODETERMINACIÓN EN EL AULA:

EL MODELO DE ENSEÑANZA Y APRENDIZAJE DE LA AUTODETERMINACIÓN

C. MUMBARDÓ-ADAM, E. VICENTE, C. GINÉ I GINÉ, J. GUARDIA-OLMOS, S. K. RALEY Y M. Á. VERDUGO

de modelos para la intervención y la promoción de las habilidades relacionadas con la autodeterminación que puedan guiar sus prácticas educativas.

En este sentido, destaca la cantidad de currículums desarrollados en inglés y diseñados específicamente para trabajar la conducta autodeterminada, frente a la escasez de recursos disponibles en español. Algunos de los programas en inglés más utilizados se recogen a continuación. El Steps to Self-Determination Curriculum (Field y Hoffman, 1996, 2000) se basa en el modelo de autodeterminación de Field y Hoffman (1994) y plasma en un programa curricular la promoción de las cinco dimensiones de las que consta dicho modelo (autoconocimiento, autoevaluación, planificación, actuación y resultados y aprendizaje). El currículum consta de una sesión teórica introductoria, un taller y 16 sesiones temáticas en las que se trabajan tanto las dimensiones como las subdimensiones de este modelo teórico para la promoción de la autodeterminación del alumno. El Self-Directed IEP, ChoiceMaker Curriculum (Martin, Huber-Marshall, Maxon, Jerman y Miller, 1996) es un programa diseñado para enseñar a los alumnos a elegir y alcanzar sus metas e incrementar las habilidades de autodeterminación necesarias para su éxito. Posee tres líneas de actuación: a) elección de metas personales, laborales y educacionales; b) expresión de dichas metas; y c) llevar a cabo la acción. El programa Next STEP (Halpern, Herr, Doren y Wolf, 2000) pretende capacitar a los alumnos para que aumenten su motivación en la etapa de transición educativa, autoevalúen sus necesidades, identifiquen sus metas personales y asuman responsabilidades sobre ellas. Además de estos tres, uno de los programas sin duda más utilizados, más extendido y con mayores pruebas que avalan su eficacia es, hasta el momento, el Self-Determination Learning Model of Instruction (SDLMI; Mithaug, Wehmeyer, Agran, Martin y Palmer, 1998; Wehmeyer, Palmer, Agran, Mithaug y Martin, 2000), que se traduce, se analiza y se presenta posteriormente en este trabajo.

Por otra parte, en España la autodeterminación está siendo muy activamente promovida por PLENA INCLUSIÓN (anteriormente FEAPS, Confederación Española de Organizaciones en favor de las Personas con Discapacidad Intelectual), así como también otras organizaciones y entidades que trabajan por y para las personas con discapacidad intelectual. Sin embargo, los esfuerzos en el diseño de estrategias de promoción de la autodeterminación, su publicación y la evaluación de su eficacia no han sido tan numerosos, destacando principalmente tres cuadernos de buenas prácticas publicados por PLENA INCLUSIÓN. En primer lugar, la "Formación en Autodeterminación para Familias” (Ponce, 2010) tiene como principal objetivo sensibilizar a las familias de jóvenes y adultos con discapacidad intelectual sobre el concepto de autodeterminación y ayudarles a entender mejor las acciones que los profesionales y servicios emprenden para apoyar la autodeterminación. En segundo lugar, el cuaderno de buenas prácticas “Autodeterminación” (Grupo de autogestores de ATZEGI, 2009) proporciona una guía para el personal de apoyo en la que se orienta sobre cómo favorecer y potenciar las oportunidades de las personas con discapacidad para practicar y desarrollar competencias de autodeterminación en la vida real. Finalmente, el programa "La autodeterminación de las personas con discapacidad intelectual” (Equipo de ASPRONA Valladolid, 2002) plasma una 
PROMOVIENDO LA AUTODETERMINACIÓN EN EL AULA:

EL MODELO DE ENSEÑANZA Y APRENDIZAJE DE LA AUTODETERMINACIÓN

C. MUMBARDÓ-ADAM, E. VICENTE, C. GINÉ I GINÉ, J. GUARDIA-OLMOS, S. K. RALEY Y M. Á. VERDUGO

experiencia real, llevada a cabo en ASPRONA, Valladolid, y que puede servir como guía para mejorar las prácticas profesionales en el apoyo a la autodeterminación de las personas con discapacidad.

El presente artículo pretende dos objetivos fundamentales: (1) presentar la versión traducida y adaptada al español del Self-Determined Learning Model of Instruction (Mithaug et al., 1998; Wehmeyer et al., 2000) (Modelo de Enseñanza y Aprendizaje de la Autodeterminación) y (2) presentar algunos de los estudios realizados en Estados Unidos que han hecho uso del modelo y avalan su eficacia, analizando las características asociadas a su implementación. En definitiva, se pretende dar a conocer una herramienta para promover la autodeterminación del alumnado que podría ser aplicable en el contexto español para apoyar a los profesionales educativos, junto a una presentación de las características y eficacia demostrada por el modelo.

\section{El Modelo de Enseñanza y Aprendizaje de la Autodeterminación}

\subsection{Estructura e implementación}

El Modelo de Enseñanza y Aprendizaje de la Autodeterminación (Mithaug et al., 1998; Wehmeyer et al., 2000) es una herramienta cuya eficacia ha sido demostrada para la enseñanza de estrategias relacionadas con la autodeterminación (p. e., Finn, Getzel y McManus, 2008; Kim y Park, 2012; Wehmeyer et al., 2012; Wehmeyer et al., 2013). Permite organizar la enseñanza de dichas estrategias a través de un proceso de resolución de problemas que el alumno debe seguir para alcanzar un objetivo preestablecido en base a sus preferencias. Además, el modelo está diseñado de manera flexible para que pueda ser implementado tanto a nivel individual como con el grupo-clase y tanto en centros ordinarios como de educación especial ya que puede y debe llevarse a cabo de forma integrada en las asignaturas del currículum. Su uso es apropiado para todos los estudiantes, con y sin discapacidad, siempre que se provean los apoyos individualizados en función de las necesidades del estudiante, en línea con los principios de los Sistemas de Apoyo Multinivel, un enfoque para la organización de los apoyos curriculares, conductuales y socioemocionales en el aula para todos los estudiantes (Sailor, 2009). Este enfoque se basa en un modelo de tres niveles de apoyos e intervenciones educativas. El primer nivel de apoyos tiene un carácter universal y tiene como finalidad poder ofrecer actividades educativas en las que todos los estudiantes puedan participar con los apoyos necesarios dentro del aula. El segundo nivel se dirige a aquellos alumnos que requieren apoyos adicionales para el logro de determinados objetivos contemplados en el currículo. En el tercer nivel se atienden las necesidades educativas de determinados alumnos que precisan el concurso de apoyos individualizados y más intensos para poder tener éxito en sus objetivos educativos. Así, en línea con estudios más recientes (Shogren, Wehmeyer y Lane, 2016), la aplicación de este modelo en el primer nivel permite que los alumnos disfruten de oportunidades explícitas para el desarrollo y la práctica de las estrategias relacionadas con la resolución de problemas y el aprendizaje autónomo y autodirigido, entre otros, 
PROMOVIENDO LA AUTODETERMINACIÓN EN EL AULA:

EL MODELO DE ENSEÑANZA Y APRENDIZAJE DE LA AUTODETERMINACIÓN

C. MUMBARDÓ-ADAM, E. VICENTE, C. GINÉ I GINÉ, J. GUARDIA-OLMOS, S. K. RALEY Y M. Á. VERDUGO

bajo una instrucción explícita y mediada por el educador (Wehmeyer, Palmer, Agran, Mithaug y Martin, 2000).

La implementación del modelo consta de tres fases: establecer un objetivo (Figura 1), actuar (Figura 2) y ajustar el objetivo o el plan (Figura 3), en las que el alumno sigue un proceso de resolución de problemas. El objetivo de la primera fase radica en que el alumno establezca un objetivo a conseguir que puede estar relacionado tanto con sus aprendizajes más académicos y curriculares, como con el aprendizaje de otras habilidades (por ejemplo, socioemocionales o relacionadas con el trabajo) que sean prioritarias para él. Esta fase puede diferir en gran manera de alumno a alumno, dado que entrará en juego, en primer lugar, el reconocimiento de sus propias necesidades e intereses y, en segundo lugar, la priorización de éstas para centrar el trabajo en una. El educador puede dar apoyo al alumno guiándole (utilizando las cuatro preguntas preestablecidas en el modelo) hacia la identificación del problema o la necesidad a trabajar; cuáles son sus habilidades o conocimientos previos relacionados con el objetivo a conseguir, y qué deberá hacer para conseguirlo. En este sentido, en la segunda fase el alumno deberá especificar el plan de acción que le va a llevar a conseguir su objetivo y específicamente: (1) pensar en las acciones que deberá realizar, (2) las posibles barreras con las que se puede encontrar y cómo las podría resolver y (3) de qué apoyos va a requerir para lograr su objetivo. Después de anticipar estos puntos, el alumno llevará a cabo su plan para acabar evaluándolo en la última fase del modelo. En esta tercera fase, el alumno evalúa el conjunto de las acciones realizadas para conseguir el objetivo marcado, los obstáculos sobrevenidos y los aprendizajes alcanzados. Deberá también valorar si ha logrado su objetivo tal y como preveía, y, en caso negativo, qué ajustes debe hacer en su plan para llegar a su objetivo.

En resumen, en cada una de las fases el modelo contempla cuatro preguntas que guían al alumno hacia el logro del objetivo marcado en cada fase. Cada una de estas preguntas está orientada a que el alumno identifique: (1) el problema, (2) sus principales soluciones, (3) los obstáculos a los que uno se puede enfrentar en función de cada posible solución y (4) las consecuencias de cada solución propuesta con apoyo del educador si es necesario. El modelo plantea igualmente una serie de objetivos del educador con cada pregunta y los posibles apoyos educativos que podría necesitar el alumno en cada fase (p. e., evaluación y conocimiento de los propios intereses y capacidades, instrucción en toma de decisiones, resolución de problemas y establecimiento de objetivos). Cabe señalar que las preguntas dirigidas al alumno se han diseñado para que el educador pueda adaptarlas en función de las necesidades de sus alumnos. En este sentido, al empezar a trabajar con el modelo se deben dar los apoyos necesarios para una comprensión fidedigna de las preguntas que guían el proceso en cada fase. Teniendo en cuenta que cada fase ilustra un problema a resolver, los alumnos deben ir respondiendo y avanzando por las cuatro preguntas que guían el proceso a fin de establecer objetivos que respondan a sus necesidades en la primera fase (Figura 1), establecer en la segunda fase planes para lograr dichos objetivos y actuar (Figura 2) $y$, finalmente, en la tercera fase evaluar la consecución de estos objetivos, así como la necesidad de ajustar los planes para lograr estos objetivos (Figura 3) (Wehmeyer, 2003). Dicho proceso se acompaña de una serie de objetivos dirigidos al educador, así 
PROMOVIENDO LA AUTODETERMINACIÓN EN EL AULA:

EL MODELO DE ENSEÑANZA Y APRENDIZAJE DE LA AUTODETERMINACIÓN

C. MUMBARDÓ-ADAM, E. VICENTE, C. GINÉ I GINÉ, J. GUARDIA-OLMOS, S. K. RALEY Y M. Á. VERDUGO

como de un listado de apoyos educativos para que, aunque el profesional adapte sus acciones educativas en función de sus alumnos, pueda disponer de unas pautas guiadas de acción que le ayuden a conseguir sus objetivos de enseñanza.

Figura 1. Fase 1 del Modelo de Enseñanza y Aprendizaje de la Autodeterminación (traducido y adaptado de Wehmeyer et al., 2000)

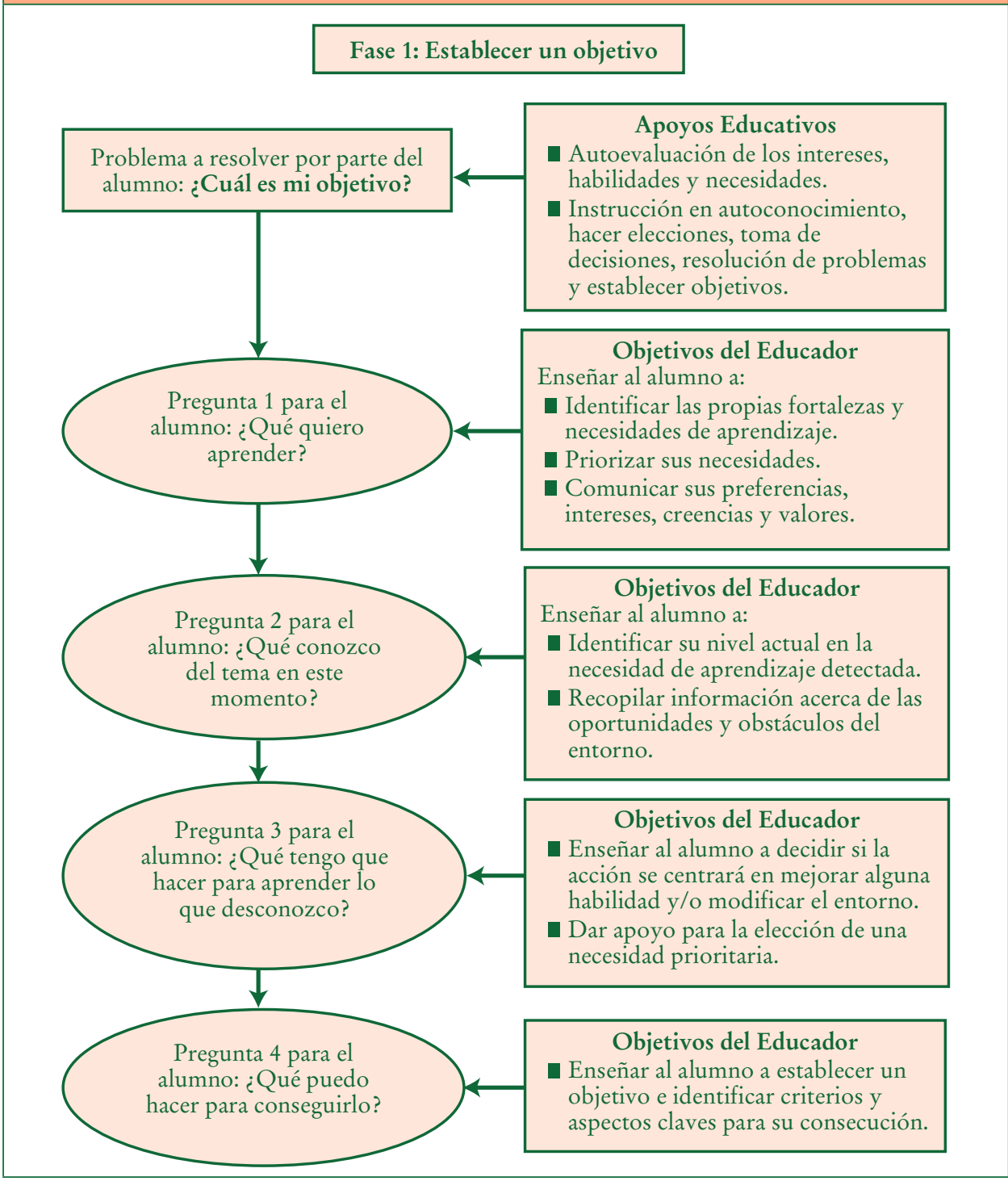

(C) Ediciones Universidad de Salamanca / CC BY-NC-ND

Siglo Cero, vol. 48 (2), n. ${ }^{\circ}$ 262, 2017, abril-junio, pp. 41-59

$$
-47-
$$


Figura 2. Fase 2 del Modelo de Enseñanza y Aprendizaje de la Autodeterminación (traducido y adaptado de Wehmeyer et al., 2000)

\section{Fase 2: Actuar}

Problema a resolver por parte del alumno: ¿Cuál es mi plan?

\section{Programación.}

- Autoaprendizaje y autosupervisión.

- Regulación con señal antecedente.

- Instrucción en la toma de decisiones y en la resolución de problemas.

- Enseñanza habilidades comunicativas.

- Estrategias para el logro de objetivos.

- Instrucción en autodefensa y asertividad.

\section{Objetivos del Educador}

Pregunta 5 para el alumno: ¿Qué puedo aprender de nuevo?



Enseñar al alumno a autoevaluar el estado actual del objetivo/necesidad de aprendizaje definido.

\section{Objetivos del Educador}

Enseñar al alumno a determinar el plan de acción para conseguir el objetivo definido, en base al estado actual.

\section{Objetivos del Educador}

Colaborar con el alumno para identificar las estrategias de aprendizaje más apropiadas.

- Enseñar estrategias de aprendizaje autodirigido y apoyar en su implementación.

- Proporcionar instrucción, previamente acordada, dirigida por el educador.

\section{Objetivos del Educador}

Enseñar al alumno a:

- Identificar su nivel actual en la necesidad de aprendizaje detectada.

- Implementar el plan de acción y autoevaluar su progreso. 
Figura 3. Fase 3 del Modelo de Enseñanza y Aprendizaje de la Autodeterminación (traducido y adaptado de Wehmeyer et al., 2000)

Fase 3: Ajustar el objetivo o el plan

Problema a resolver por parte del alumno: ¿Qué he aprendido?



Apoyos Educativos

Estrategias de autoevaluación.

Instrucción en la toma de decisiones, la resolución de problemas y en el establecimiento de objetivos.

- Estrategias de autorrefuerzo.

- Estrategias de autorregistro.

- Autosupervisión.

\section{Objetivos del Educador}

Enseñar a los alumnos a autoevaluar su progreso hacía el logro de su objetivo.

\section{Objetivos del Educador}

- Colaborar con el alumno para comparar el progreso conseguido con los resultados deseados.

\section{Objetivos del Educador}

Dar apoyo al alumno para reevaluar el objetivo en caso de que el progreso no sea el esperado y decidir si se cambia el objetivo o no.

- Colaborar con el alumno para identificar la adecuación del plan de acción en base al objetivo revisado.

\section{Objetivos del Educador}

Enseñar al alumno a decidir si el progreso ha sido adecuado o inadecuado y si el objetivo se ha logrado. 
PROMOVIENDO LA AUTODETERMINACIÓN EN EL AULA:

EL MODELO DE ENSEÑANZA Y APRENDIZAJE DE LA AUTODETERMINACIÓN

C. MUMBARDÓ-ADAM, E. VICENTE, C. GINÉ I GINÉ, J. GUARDIA-OLMOS, S. K. RALEY Y M. Á. VERDUGO

\subsection{Usos y aplicaciones del modelo en contexto educativo}

El Modelo de Enseñanza y Aprendizaje de la Autodeterminación (Mithaug et al., 1998; Wehmeyer et al., 2000) ha demostrado ser una práctica basada en la evidencia para la promoción de la autodeterminación (Test et al., 2009). No obstante, no solo sirve para trabajar directamente la autodeterminación (p. e., Wehmeyer et al., 2012), sino también promueve las estrategias y habilidades relacionadas con la autodeterminación (p. e., resolución de problemas, autorregulación, aprendizaje autodirigido), así como mejora el acceso al currículum, la participación en contextos ordinarios o la consecución de metas académicas o de transición. Las tablas que se presentan a continuación pretenden resumir las principales características de diversos estudios que describen el uso del SDLMI en Estados Unidos, a fin de complementar la posterior descripción de los estudios y de sus resultados. Dichas características se presentan en función de los objetivos para los que se ha implementado el SDLMI: el acceso al currículum y la participación en actividades educativas (Tabla 1); la enseñanza de habilidades de resolución de problemas (Tabla 2); y el establecimiento y consecución de metas y objetivos personales (Tabla 3).

En la Tabla 1, se detallan ejemplos de estudios que se han llevado a cabo para fomentar el acceso y la participación del alumnado con discapacidad en actividades cotidianas del aula. En efecto, Agran et al. (2006, 2008 y 2010) realizaron varios estudios de caso múltiple con alumnos con necesidades de apoyo de leves a severas. Los resultados indicaron que, en todos los casos, los alumnos aumentaron su participación activa en el aula ordinaria, sobrepasando el criterio mínimo de dominio establecido, y mantuvieron dicho aprendizaje una vez terminada la intervención. Además, la validación social de la intervención, preguntando a los alumnos y maestros sobre la experiencia, fue muy positiva (Agran et al., 2006, 2010). Por su parte, Kelly y Shogren (2014) llevaron a cabo una intervención con cuatro adolescentes que presentaban conductas desafiantes para aumentar su participación en las actividades curriculares disminuyendo, a su vez, las conductas inapropiadas que se dan durante la realización de las mismas y que ineludiblemente interfieren. Después de la intervención con el SDLMI, los alumnos aumentaron su participación activa en las actividades curriculares; lograron autorregular y disminuir las conductas disruptivas no relacionadas con las actividades, y mantuvieron el aprendizaje una vez terminada la intervención. Como en Agran et al. (2006, 2010), la validación social de la intervención con el SDLMI fue muy positiva. Otras intervenciones llevadas a cabo en el aula ordinaria (Lee et al., 2008; Palmer et al., 2004) demuestran también que el trabajo con el SDLMI promueve el acceso al currículo ordinario. Finalmente, cabe destacar que adaptaciones del SDLMI a otros contextos como el familiar (Kim y Park, 2012) han demostrado ser también una herramienta eficaz de promoción y refuerzo de las habilidades trabajadas en contexto escolar. 
PROMOVIENDO LA AUTODETERMINACIÓN EN EL AULA:

EL MODELO DE ENSEÑANZA Y APRENDIZAJE DE LA AUTODETERMINACIÓN

C. MUMBARDÓ-ADAM, E. VICENTE, C. GINÉ I GINÉ, J. GUARDIA-OLMOS, S. K. RALEY Y M. Á. VERDUGO

\begin{tabular}{|c|c|c|c|c|c|c|c|c|c|c|c|}
\hline \multirow{11}{*}{ 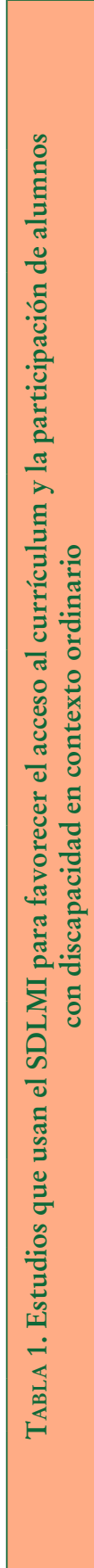 } & \multirow{4}{*}{2} & 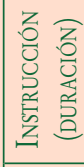 & 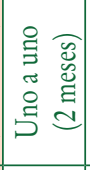 & 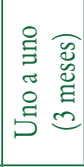 & $\begin{array}{l}0 \\
\Xi \\
\tilde{\Xi} \\
0 \\
\vdots\end{array}$ & 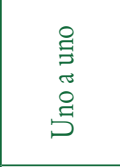 & 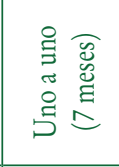 & 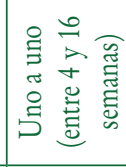 & 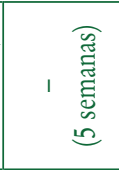 & 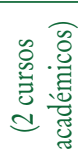 & \multirow{4}{*}{  } \\
\hline & & $\begin{array}{l}0 \\
\text { 离 } \\
\text { 至 } \\
0 \\
0\end{array}$ & 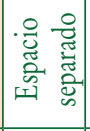 & 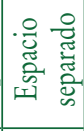 &  &  & 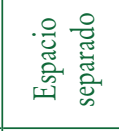 & 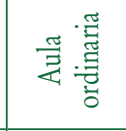 & 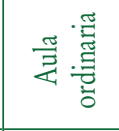 & 1 & \\
\hline & & 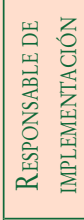 &  & 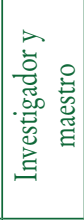 & 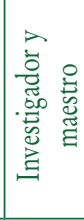 & 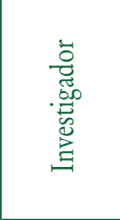 & 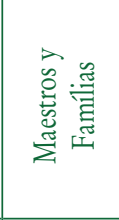 &  &  & 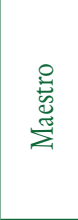 & \\
\hline & & 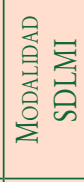 & 莺 &  &  & 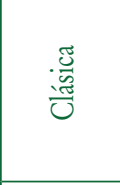 & 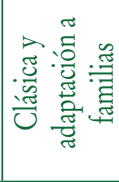 & 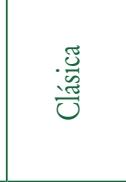 & 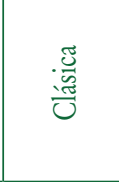 & 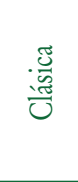 & \\
\hline &  & 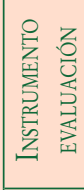 & 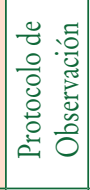 & 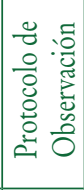 & 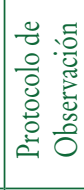 & 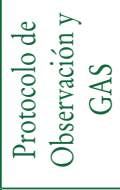 & 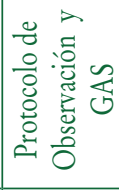 & 产这 & 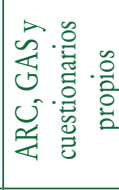 & 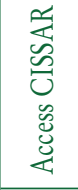 & \\
\hline & 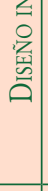 & $\stackrel{\text { }}{€}$ & 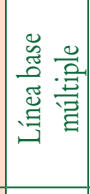 & 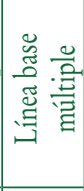 & 总 & 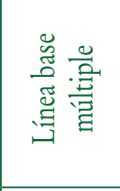 & 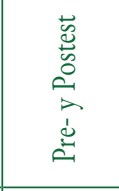 & 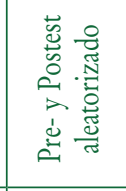 & 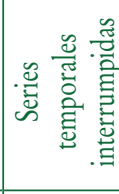 &  & \\
\hline & & 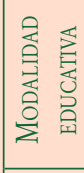 & 匀 & 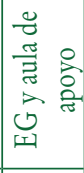 & 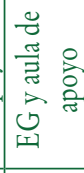 & 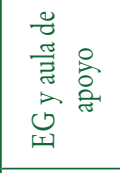 & 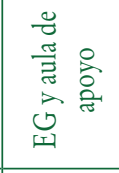 & 至 & 可 & $\begin{array}{l}0 \\
0 \\
0 \\
0 \\
0 \\
0 \\
2\end{array}$ & \\
\hline & $\begin{array}{l}\vec{Z} \\
\text { 妾 }\end{array}$ & 斊 & $\frac{n}{ \pm}$ & $\frac{n}{2}$ & $\frac{n}{ \pm}$ & $\frac{0}{\dot{1}}$ & 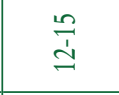 & $\frac{9}{ \pm}$ & $\frac{10}{3}$ & & \\
\hline &  & $\stackrel{\ominus}{\ominus}$ & $\vec{\nabla}$ & 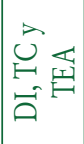 & $\begin{array}{l}0 \\
\overrightarrow{0} \\
\overrightarrow{0}\end{array}$ & 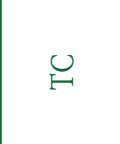 & 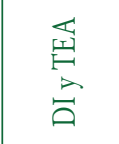 & 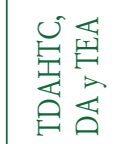 & $\bar{\imath}$ & $\stackrel{a}{a}$ & \\
\hline & & Z & $m$ & $m$ & $m$ & + & $\underset{\sim}{ \pm}$ & $\stackrel{q}{q}$ & శ & 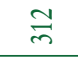 & \\
\hline & & 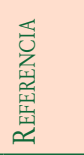 & 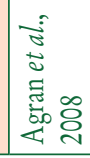 & 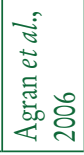 &  & 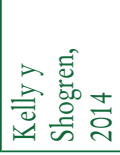 & 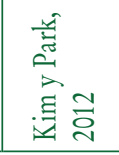 &  & 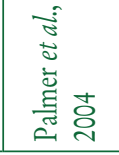 & 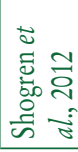 & \\
\hline
\end{tabular}

(c) Ediciones Universidad de Salamanca / CC BY-NC-ND

Siglo Cero, vol. 48 (2), n. ${ }^{\circ}$ 262, 2017, abril-junio, pp. 41-59 
PROMOVIENDO LA AUTODETERMINACIÓN EN EL AULA:

EL MODELO DE ENSEÑANZA Y APRENDIZAJE DE LA AUTODETERMINACIÓN

C. MUMBARDÓ-ADAM, E. VICENTE, C. GINÉ I GINÉ, J. GUARDIA-OLMOS, S. K. RALEY Y M. Á. VERDUGO

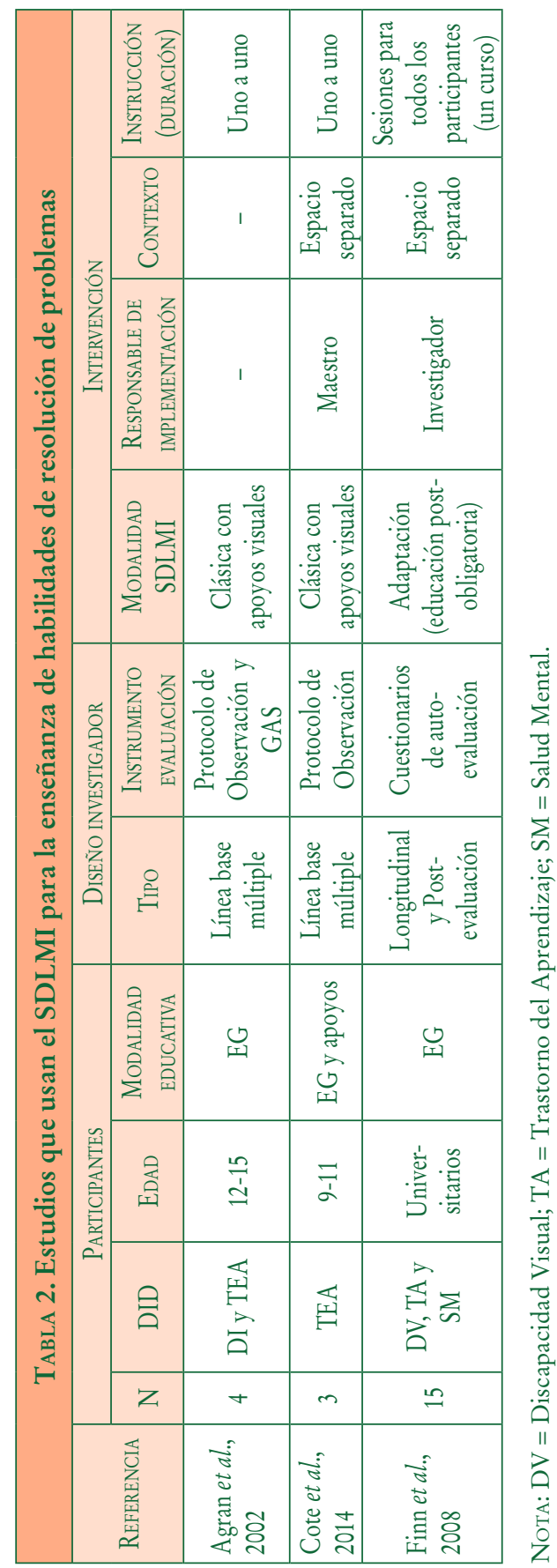

(c) Ediciones Universidad de Salamanca / CC BY-NC-ND

Siglo Cero, vol. 48 (2), n. ${ }^{\circ}$ 262, 2017, abril-junio, pp. 41-59 
PROMOVIENDO LA AUTODETERMINACIÓN EN EL AULA:

EL MODELO DE ENSEÑANZA Y APRENDIZAJE DE LA AUTODETERMINACIÓN

C. MUMBARDÓ-ADAM, E. VICENTE, C. GINÉ I GINÉ, J. GUARDIA-OLMOS, S. K. RALEY Y M. Á. VERDUGO



(c) Ediciones Universidad de Salamanca / CC BY-NC-ND

Siglo Cero, vol. 48 (2), n. ${ }^{\circ}$ 262, 2017, abril-junio, pp. 41-59 
PROMOVIENDO LA AUTODETERMINACIÓN EN EL AULA:

EL MODELO DE ENSEÑANZA Y APRENDIZAJE DE LA AUTODETERMINACIÓN

C. MUMBARDÓ-ADAM, E. VICENTE, C. GINÉ I GINÉ, J. GUARDIA-OLMOS, S. K. RALEY Y M. Á. VERDUGO

Otra de las habilidades relacionadas con la autodeterminación que se ha estudiado con la aplicación del Modelo de Enseñanza y Aprendizaje de la Autodeterminación (Mithaug et al., 1998; Wehmeyer et al., 2000) es la enseñanza de habilidades de resolución de problemas (Tabla 2). Agran et al. (2002) y Cote et al. (2014) desarrollaron intervenciones con alumnos con discapacidad intelectual y con trastorno de espectro autista, respectivamente. En ambos casos, los alumnos aprendieron estrategias de resolución de problemas para conseguir los resultados que se habían marcado en la Fase 1 del modelo, y mantuvieron dicho aprendizaje una vez terminada la intervención. Además, los resultados de dichas intervenciones sobrepasaron las expectativas de los maestros (Agran et al., 2002). Tanto Agran et al. (2002) como Cote et al. (2014) utilizaron el modelo en niños (entre 12 y 15 y entre 9 y 11 años, respectivamente), sin embargo, cabe destacar el estudio de Finn et al. (2008) en el que adaptaron el SDLMI para alumnos universitarios con necesidades de apoyo específicas (discapacidad visual, trastorno de aprendizaje o problema de salud mental). Todos ellos reportaron mejoras en sus habilidades de resolución de problemas, así como una mejora en la autoconfianza.

Finalmente, el establecimiento y consecución de objetivos constituye otro elemento relacionado con la conducta autodeterminada que se ha trabajado y promovido con el SDLMI (Tabla 3), casi siempre con jóvenes adolescentes, aunque también con niños de edades más tempranas (Palmer y Wehmeyer, 2003). Concretamente, Kleinert, Harrison, Dueppen, Mills y Trailor (2014) analizaron los datos post-hoc de un estudio longitudinal realizado durante un curso académico entero en el que los estudiantes debían marcarse objetivos concretos y desarrollarlos con el apoyo del modelo y la instrucción por parte de los educadores. Del total de objetivos iniciales establecidos, se alcanzaron más del $70 \%$ y se hicieron progresos en los que no se alcanzaron totalmente. En relación a la tipología de objetivos marcados, aunque la mayoría de los estudios revisados incluían objetivos de tipo educativo o curricular, resulta interesante destacar que el modelo ha resultado ser también de utilidad en objetivos relacionados con la disminución de conductas problemáticas (Mazzotti, Test y Wood, 2012; Mazzotti, Wood, Test y Fowler, 2012) o relacionados con el aprendizaje de habilidades que favorecen el desempeño de la persona en futuros entornos laborales (McGlashing-Johnson et al., 2003). Otros objetivos de trabajo han sido la enseñanza de habilidades como la autorregulación y el aprendizaje autodirigido. Agran et al. (2001) enseñaron a seis alumnos con discapacidad a monitorizar, evaluar y reforzar su propio avance en los objetivos marcados mediante el modelo, optimizando sus habilidades de aprendizaje autodirigido.

\section{Conclusiones}

En el presente trabajo se ha presentado la versión traducida de una herramienta de promoción de la autodeterminación: el Modelo de Enseñanza y Aprendizaje de la Autodeterminación (Mithaug et al., 1998; Wehmeyer et al., 2000), para que pueda ser aplicada en contexto español. A su vez, se han presentado los estudios que describen 
PROMOVIENDO LA AUTODETERMINACIÓN EN EL AULA:

EL MODELO DE ENSEÑANZA Y APRENDIZAJE DE LA AUTODETERMINACIÓN

C. MUMBARDÓ-ADAM, E. VICENTE, C. GINÉ I GINÉ, J. GUARDIA-OLMOS, S. K. RALEY Y M. Á. VERDUGO

el uso de dicha herramienta para promover estrategias relacionadas con la conducta autodeterminada. Dichos estudios tienen su fundamento en investigaciones diseñadas para comprobar la eficacia y el impacto de la intervención con el SDLMI en distintos contextos y para distintos objetivos. Los diseños de la mayoría de ellos eran principalmente estudios de línea base múltiple, con muestras reducidas y en los que se registra la evolución del alumnado mediante la observación sistemática (p. e., Cote et al., 2014); aunque también se han realizado algunos con un mayor número de participantes (Shogren et al., 2012). Aunque el SDLMI se ha usado mayoritariamente con alumnos con discapacidad intelectual (p. e., Agran et al., 2008), es importante señalar que, aunque menos frecuente, también se ha utilizado el modelo con alumnos con otro tipo de dificultades o necesidades educativas. En concreto, se ha implementado dicha estrategia de intervención con alumnos con trastorno del espectro del autismo (p. e., Cote et al., 2014), con trastorno de la conducta (p. e., Kelly y Shogren, 2014) y alumnos con dificultades de aprendizaje y trastorno por déficit de atención y/o hiperactividad (p. e., Lee et al., 2008); en menor medida también con alumnos con discapacidad auditiva, alumnos con problemas de salud mental (Agran et al., 2001) y alumnos con altas capacidades (Palmer y Wehmeyer, 2003). La intervención con el SDLMI se ha llevado a cabo principalmente con adolescentes y jóvenes de entre 10 y 21 años, aunque también se ha probado su eficacia con alumnos de 5 a 9 años (Palmer y Wehmeyer, 2003) e incluso con universitarios (Finn et al., 2008). Se ha comprobado también la eficacia del instrumento tanto con la aplicación clásica del modelo, como con versiones adaptadas. En efecto, en algunos estudios se ha completado el SDLMI con apoyos visuales para favorecer su utilización y comprensión por parte del alumnado (p. e., Agran et al., 2002), en otros se ha trabajado con una versión computerizada del mismo (Mazzotti, Test et al., 2012) y también se ha adaptado el modelo al contexto familiar (Kim y Park, 2012).

Así pues, destaca la cantidad de estudios en el contexto americano en los que el uso del SDLMI ha demostrado fomentar la autodeterminación (Wehmeyer, Shogren, Palmer, Williams-Diehm, Little y Boulton, 2012), tal y como señalan los resultados de los estudios anteriormente resumidos. En concreto, su utilidad se ha estudiado en relación a la enseñanza de habilidades como la autorregulación, la resolución de problemas (Cote et al., 2014) y el establecimiento de objetivos (Kleinert et al., 2014), estrategias cuyo fomento tiene un impacto positivo en la autodeterminación de la persona, tenga discapacidad o no. Todo contexto educativo debe así promover oportunidades para el desarrollo de dichas habilidades y, en el caso de alumnos con discapacidad, este aprendizaje debe darse prioritariamente en entornos inclusivos (Shogren, McCart, Lyon y Sailor, 2015), a fin de favorecer un aprendizaje enraizado en el currículum educativo. Si bien el aprendizaje de estas estrategias en contexto escolar es beneficioso para todos los alumnos (Shogren, Lopez, Wehmeyer, Little y Pressgrove, 2006; Shogren, Wehmeyer y Lane, 2016), en la mayoría de estudios anteriormente presentados, como ejemplos de aplicaciones del SDLMI, las intervenciones se llevan a cabo con un alumno con discapacidad en el tercer nivel del sistema multinivel de apoyos (p. e., Agran et al., 2006) o bien con un grupo de alumnos con necesidades de apoyo en el segundo nivel (p. e., Finn et al., 2008). Además, aunque en la gran mayoría de estudios 
PROMOVIENDO LA AUTODETERMINACIÓN EN EL AULA:

EL MODELO DE ENSEÑANZA Y APRENDIZAJE DE LA AUTODETERMINACIÓN

C. MUMBARDÓ-ADAM, E. VICENTE, C. GINÉ I GINÉ, J. GUARDIA-OLMOS, S. K. RALEY Y M. Á. VERDUGO

analizados los participantes están escolarizados en escuelas ordinarias, la instrucción con el SDLMI se suele dar de uno a uno, es decir, de maestro o investigador al alumno objeto de la intervención y principalmente en ambientes separados del aula ordinaria.

Estudios recientes (Mumbardó-Adam, Shogren, Guàrdia-Olmos y Giné, 2017; Shogren, Wehmeyer y Lane, 2016) ponen de relieve la necesidad de avanzar hacia la promoción de iniciativas de enseñanza y oportunidades de aprendizaje de la autodeterminación que se den por igual para todos los estudiantes en el primer nivel del sistema de apoyos, de forma que se reserva la instrucción en los otros dos niveles para aquellos alumnos que requieran apoyos adicionales y/o intensivos (Lane et al., 2007); en definitiva, se apuesta así por unas prácticas educativas que ofrecen a todos los alumnos la oportunidad de aprender unas estrategias y habilidades esenciales para su desarrollo en contextos postescolares. Así, en el futuro, las investigaciones deberán avanzar hacia la promoción e instrucción de la autodeterminación en los contextos naturales para todos los estudiantes, con o sin discapacidad. En esta línea, el acceso de los profesionales a programas e instrumentos rigurosos de enseñanza de la autodeterminación permitirá reducir la brecha que actualmente separa en nuestro contexto la evaluación de la intervención psicoeducativa en materia de autodeterminación. Los recursos y esfuerzos invertidos para establecer medidas válidas y fiables del constructo (p. e., Verdugo et al., 2015) permiten dotar al profesional del conocimiento sobre las habilidades de la persona en relación a su autodeterminación. Dichos conocimientos, a su vez, establecen los fundamentos de los objetivos individualizados de intervención. Por ello, resulta crítico poder disponer de investigaciones que faciliten el tránsito de la evaluación a la intervención, guiadas, entre otros, por el modelo que se acaba de presentar. Así, una futura línea de investigación debe enfocarse, sin duda, en aportar evidencias empíricas que avalen la eficacia del modelo en contexto español, $\mathrm{y}$ en los tres niveles del sistema de apoyos multinivel.

\section{Referencias bibliográficas}

Agran, M., Blanchard, C., Wehmeyer, M. L. y Hughes, C. (2001). Teaching students to self-regulate their behavior: the differential effects of students vs. teacher-delivered reinforcement. Research in Developmental Disabilities, 22, 319-332.

Agran, M., Blanchard, C., Wehmeyer, M. L. y Hughes, C. (2002). Increasing the problemsolving skills of students with developmental disabilities participating in general education. Remedial and Special Education, 23 (5), 279-288.

Agran, M., Cavin, M., Wehmeyer, M. L. y Palmer, S. (2006). Participation of students with moderate to severe disabilities in the general curriculum: The effects of the Self-Determined Learning Model of Instruction, Research E Practice for Persons with Severe Disabilities, 31 (3), 230-241. doi: 10.1177/154079690603100303.

Agran, M., Cavin, M., Wehmeyer, M. L. y Palmer, S. (2010). Promoting active engagement in the general education curriculum for students with cognitive disabilities. Education and Training in Autism and Developmental Disabilities, 45 (2), 163-174.

Agran, M. y Wehmeyer, M. L. (2000). Promoting transition goals and self-determination through student self-directed learning: The Self-Determined Learning Model of Instruction. 
PROMOVIENDO LA AUTODETERMINACIÓN EN EL AULA:

EL MODELO DE ENSEÑANZA Y APRENDIZAJE DE LA AUTODETERMINACIÓN

C. MUMBARDÓ-ADAM, E. VICENTE, C. GINÉ I GINÉ, J. GUARDIA-OLMOS, S. K. RALEY Y M. Á. VERDUGO

Education and Training in Mental Retardation and Developmental Disabilities, 35 (4), 351 364. doi: 10.1177/0885728808317656.

Agran, M., Wehmeyer, M. L., Cavin, M. y Palmer, S. (2008). Promoting student active classroom participation skills through instruction to promote self-regulated learning and selfdetermination. Career Development for Exceptional Individuals, 31 (2), 106-114.

Cote, D. L., Jones, V. L., Barnett, C., Pavelek, K., Nguyen, H. y Sparks, S. L. (2014). Teaching problem solving skills to elementary age students with autism. Education and Training in Autism and Developmental Disabilities, 49 (2), 189-199.

EQUIPO DE PROFESIONALES ASPRONA (2002). La autodeterminación de las personas con discapacidad intelectual. Madrid: FEAPS, Cuadernos de Buenas Prácticas.

Field, S. y Hoffman, A. (1994). Development of a model for self-determination. Career Development for Exceptional Individuals, 17 (2), 159-169.

Field, S. y Hoffman, A. (1996). Steps to self-determination: A curriculum to belp adolescents learn to achieve their goals. Austin, TX: Pro-Ed.

Field, S. y Hoffman, A. (2000). Steps to self-determination idea book. Detroit, MI: Wayne State University.

Finn, D., Getzel, E. E. y McManus, S. (2008). Adapting the Self-Determined Learning Model of Instruction for college Students with disabilities. Career Development for Exceptional Individuals, 31 (2), 85-93. doi: 10.1177/0885728808318327.

Grupo de autogestores de ATZEGI (2009). Autodeterminación. Madrid: FEAPS, Cuadernos de Buenas Prácticas.

Halpern, A. S., Herr, C. M., Doren, B. y Wolf, N. H. (2000). NEXT S.T.E.P. Student Transition and Educational Planning (2. ${ }^{\mathrm{a}}$ ed.). Austin: PRO-ED.

Kelly, J. R. y Shogren, K. A. (2014). The impact of teaching self-determination skills on the on-task and off-task behaviors of students with emotional and behavioral disorders. Journal of Emotional and Behavioral Disorders, 22 (1), 27-40.

KIM, N. H. y PARK, J. (2012). The effects of the family-involved SDLMI on academic engagement and goal attainment of middle school students with disabilities who exhibit problem behavior. International Journal of Special Education, 27 (1), 117-127.

Kiresuck, T. J., Smith, A. y Cardillo, J. (1994). Goal attainment scaling: Applications, theory, and measurement. Hillsdale, NJ: Lawrence Erlbaum.

Kleinert, J. O., Harrison, E., Dueppen, B. M., Mills, K. R. y Trailor, A. M. (2014). Selfdetermined goal selection and planning by students with disabilities across grade bands and disability categories. Education and Training in Autism and Developmental Disabilities, 49 (3), 464-477.

Lane, K. L., Rogers, L. A., Parks, R. J., Weisenbach, J. L., Mau, A. C., Merwin, M. T. y BERgMAN, W. A. (2007). Function-based interventions for students who are nonresponsive to primary and secondary prevention efforts: Illustrations at the elementary and middle school levels. Journal of Emotional and Behavioral Disorders, 15, 169-183. doi: 10.1177/10 634266070150030401.

Lee, S-H., Wehmeyer, M. L., Palmer, S. B., Soukup, J. H. y Little, T. D. (2008). Self-determination and access to the general education curriculum. The Journal of Special education, 42 (2), 91-107. doi: 10.1177/0022466907312354.

Martin, J., Huber-Marshall, L., Maxton, L., Jerman, P. y Miller, T. L. (1996). The self-directed IEP. Longmont, CO: Sopris West.

MazzotTi, V. L., Test, D. W. y Wood, C. L. (2012). Effects of multimedia goal-setting on students' knowledge of the Self-Determined Learning Model of Instruction and disruptive behavior. Journal of Positive Behavior Interventions, 15 (2), 90-102.

(C) Ediciones Universidad de Salamanca / CC BY-NC-ND

Siglo Cero, vol. 48 (2), n. ${ }^{\circ}$ 262, 2017, abril-junio, pp. 41-59 
PROMOVIENDO LA AUTODETERMINACIÓN EN EL AULA:

EL MODELO DE ENSEÑANZA Y APRENDIZAJE DE LA AUTODETERMINACIÓN

C. MUMBARDÓ-ADAM, E. VICENTE, C. GINÉ I GINÉ, J. GUARDIA-OLMOS, S. K. RALEY Y M. Á. VERDUGO

Mazzotti, V. L., Wood, C. L., Test, D. W. y Fowler, C. H. (2012). Effects of computerassisted instruction on students' knowledge of the Self-Determined Learning Model of Instruction and disruptive behavior. The Journal of Special Education, 45 (4), 216-226.

McGlashing-Johnson, J., Agran, M., Sitlington, P. y Cavin, M. (2003). Enhancing the job performance of youth with moderate to severe cognitive disabilities using the Self-Determined learning Model of Instruction. Research E Practice for Persons with Severe Disabilities, 28 (4), 194-204. doi: 10.2511/rpsd.28.4.194.

Mithaug, D. E., Wehmeyer, M. L., Agran, M., Martin, J. y Palmer, S. B. (1998). The SelfDetermined Learning Model of Instruction: Engaging students to solve their learning problems. En M. L. WehmeYer y D. J. SAnds (Eds.), Making it happen: Student involvement in educational planning, decision-making and instruction (pp. 299-328). Baltimore: Paul H. Brookes Publishers.

Mumbardó-Adam, C., Shogren, K. A., Guàrdia-Olmos, J. y Giné, C. (2017). Contextual predictors of self-determined actions in students with and without intellectual disability. Psychology in the Schools, 54 (2), 115-2019. doi: 10.1002/pits.21987.

Nowicki, S. y Duke, M. P. (1974). A locus of control scale for non-college as well as college adults. Journal of Personality Assessment, 38, 136-137.

Palmer, S. B. y Wehmeyer, M. L. (2003). Promoting self-determination in early elementary school: Teaching self-regulated problem-solving and goal-setting Skills. Remedial and Special Education, 24 (2), 115-126. doi: 10.1177/07419325030240020601.

Palmer, S. B., Wehmeyer, M. L., Gipson, K. y Agran, M. (2004). Promoting access to the general education curriculum by teaching self-determination skills. Exceptional Children, 70 (4), 427-439.

Palmer, S. B., Wehmeyer, M. L. y Shogren, K. A. (2016). The development of self-determination. En M. L. Wehmeyer, K. A. Shogren, T. Little y S. López (Eds.), Handbook on the development of self-determination. New York: Springer.

PONCE, A. (2010). Formación en autodeterminación para familias. Madrid: FEAPS, Cuadernos de Buenas Prácticas.

SAILOR, W. S. (2009). Making RTI work: How schools are reforming education through schoolwide response-to-intervention. Wiley.

Shogren, K. A., López, S. J., Wehmeyer, M. L., Little, T. D. y Pressgrove, C. L. (2006). The role of positive psychology constructs in predicting life satisfaction in adolescents with and without cognitive disabilities: An exploratory study. Journal of Positive Psychology, 1, 3752. doi: 10.1080/17439760500373174.

Shogren, K. A., McCart, A. B., Lyon, K. J. y Sailor, W. S. (2015). All means all: Building knowledge for inclusive schoolwide transformation. Research and Practice for Persons with Severe Disabilities, 40 (3), 173-191. doi: 10.1177/1540796915586191.

Shogren, K. A., Palmer, S. B., Wehmeyer, M. L., Williams-Diehm, K. y Little, T. D. (2012). Effect of intervention with the Self-Determined Learning Model of Instruction on access and goal attainment. Remedial and Special Education, 33 (5), 320-330.

Shogren, K. A., Wehmeyer, M. L. y Lane, K. L. (2016). Embedding interventions to promote self-determination within multi-tiered systems of supports. Exceptionality.

Shogren, K. A., Wehmeyer, M. L., Little, T. D., Forber-Pratt, A. J., Palmer, S. B. y Seo, H. (2015). Preliminary validity and reliability of scores on the Self-Determination InventoryStudent Report version. Career for Development and Transition for Exceptional Individuals, 50 (3), 251-263. doi: 10.1177/2165143415594335.

(C) Ediciones Universidad de Salamanca / CC BY-NC-ND

Siglo Cero, vol. 48 (2), n. ${ }^{\circ}$ 262, 2017, abril-junio, pp. 41-59 
PROMOVIENDO LA AUTODETERMINACIÓN EN EL AULA:

EL MODELO DE ENSEÑANZA Y APRENDIZAJE DE LA AUTODETERMINACIÓN

C. MUMBARDÓ-ADAM, E. VICENTE, C. GINÉ I GINÉ, J. GUARDIA-OLMOS, S. K. RALEY Y M. Á. VERDUGO

Shogren, K. A., Wehmeyer, M. L., Palmer, S. B., Forber-Pratt, A. J., Little, T. D. y Lopez, S. (2015). Causal agency theory: reconceptualizing a functional model of self-determination. Education and Training in Autism and Developmental Disabilities, 50 (3), 251-263.

Shogren, K. A., Wehmeyer, M. L., Palmer, S. B., Rifenbark, G. G. y Little, T. D. (2015). Relationships between self-determination and postschool outcomes for youth with disabilities. Journal of Special Education, 53, 30-41. doi: 10.1177/0022466913489733.

Test, D. W., Mazzotti, V. L., Mustian, A. L., Fowler, C. H., Kortering, L. y Kohler, P. (2009). Evidence-based secondary transition predictors for improving postschool outcomes for students with disabilities. Career Development for Exceptional Individuals, 32 (3), 160-181. doi: 10.1177/0885728809346960.

Verdugo, M. Á., Vicente, E., Gómez-Vela, M., Fernández, R., Wehmeyer, M. L., Badia, M., González-Gil, F. y Calvo, M. I. (2015). Escala ARC-INICO de Evaluación de la Autodeterminación: Manual de aplicación y corrección. Salamanca: INICO.

Vicente, E., Guillén, V. y Verdugo, M. Á. (2014). La autodeterminación a lo largo de la vida de las personas con discapacidad. Revisión de recursos para profesionales. En D. Wojcik, F. Cruz y M. Tejedor (Coords.), Congreso Internacional de Servicios Sociales y Sociosanitarios: Retos y perspectivas 2020 para la protección y el desarrollo social (p. 22). Palencia: Ayuntamiento de Palencia.

Wehmeyer, M. L. (2003). A functional theory of self-determination: Definition and categorization. En M. L. Wehmeyer, B. Abery, D. Mithaug y R. Stancliffe (Eds.), Theory in self-determination: Foundations for educational practice (pp. 174-181). Springfield: Thomas Publishing Co.

Wehmeyer, M. L. y Kelchner, K. (1995). The Arc's self-determination scale. Silver Springs, MD: The Arc of the United States.

Wehmeyer, M. L., Palmer, S., Agran, M., Mithaug, D. y Martin, J. (2000). Promoting causal agency: The self-determined learning model of instruction. Exceptional Children, 66, 439-453.

Wehmeyer, M. L., Palmer, S. B., Shogren, K. A., Williams-Diehm, K. y Soukup, J. H. (2013). Establishing a causal relationship between intervention to promote self-determination and enhanced student self-determination. The Journal of Special Education, 46 (4), 195-210. doi: $10.1177 / 0022466910392377$.

Wehmeyer, M. L., Peralta, F., Zulueta, A., González Torres, M. C. y Sobrino, A. (2006). Escala de autodeterminación personal ARC Instrumento de valoración y guia de aplicación: Manual Técnico de la adaptación española. Madrid: CEPE.

Wehmeyer, M. L., Shogren, K. A., Palmer, S. B., Williams-Diehm, K. L., Little, T. D. y Boulton, A. (2012). The impact of the Self-Determined Learning Model of Instruction on student self-determination. Exceptional Children, 78 (2), 135-153.

Wolman, J. M., Campeau, P. L., Dubois, P. A., Mithaug, D. E. y Stolarski, V. S. (1994). Air Self-Determination Scale and User Guide. Columbia: American Institutes for Research. 